\title{
Research on Prediction Model of Stock Price Based on LM-BP Neural Network
}

\author{
LI Feng \\ College of International Business, Shenyang Normal \\ University \\ Shenyang, China \\ email:lifeng670312@sina.com
}

\begin{abstract}
LM (Levenberg-Marquardt) algorithm is an optimization algorithm for the overall situation which is appropriate for neural network training. It is assumed that standard BP neural network tends to reach the local minimum instead of the optimization of the whole, demand much more training resulting in the low learning efficiency and low rate of convergence. At the same time, it seems easily to forget former sample. To solve the above disadvantages, this paper intends to form prediction model of stock price based on LM- BP neural network to forecast stock price, as well as compared with the prediction result carried out by standard BP neural network. At the end, this paper achieves the satisfactory result.
\end{abstract}

Keywords- LM-BP neural network, stock price, prediction

\section{PREFACE}

BP neural network is the most typical algorithm in neural network. BP neural network is a multilayer feedforward neural network. If activation function is $S$ and output quantity is continuous variable from 0 to $1, \mathrm{BP}$ network could realize random non-linear mapping from input to output. The basic principle of standard BP learning algorithm adapts least square method, applying gradient search to make sum of error-square between actual output quantity and expected output quantity possibly reaching minimum. BP neural network is a process to correct weight coefficient as well as to spread error backward. Actually this process involves basically to spreading orientation, forward and backward. The first one is forward-propagating. Sample enters through the input layer, refined by hidden units step-by-step and then reaches output layer. Output of neuron in each individual could simply influence its down neuron gradually and at last reaches output layer. If there is error between network output value and expected value, it will go to the opposite process. The second one is backward propagating. Error signal turns to backward propagating from the original orientation, following negative gradient of error function to correct weight coefficient of each layer gradually to have the minimum error function. Here we may conclude that BP algorithm is a searching algorithm based on gradient. Its prominent superiority includes the three layer feedforward neural network of non-linear transfer function which makes it possible to approach any non-liner function at any accuracy. However, it is widely recognized that BP has some intrinsic fault. It is assumed that standard BP neural network tends to reach the local minimum instead of the optimization on the whole, appears to need much more training resulting in the low learning efficiency and low rate of convergence, at the same time, it seems easily to forget former sample. To solve the above disadvantages, this paper intends to form prediction model of stock price based on LM- BP neural network to forecast stock price, as well as compares with the prediction result carried out by standard BP neural network, hopefully at the end, to reach the satisfactory result.

\section{PREDICTION METHOD OF STOCK PRICE BASED ON LM-BP NEURAL NETWORK}

\section{A. LM algorithm}

LM algorithm is an optimization algorithm for the overall situation which can be applied to neural network training appropriately. Standard BP neural network calculates each sample both forward and backward oriented to get modified value related to weight vector $\mathrm{W}$ which then would be modified. On the contrary, LM uses all the samples to get modified value of $\mathrm{W}$, and then to modify W. One of the basic LM algorithms is Gauss Newton algorithm. LM algorithm is the up-grated algorithm.

\section{B. LM-BP network algorithm}

When structure of network is fixed and then chooses well the excitation function of the hidden neuron, the basic element of non-linear formula $y=f(X, W)$ is completed. On the basis of $y=f(X, W)$, we could get the formula $\left(\frac{\partial f(X, W)}{\partial W}\right)$.So far, we have completed the training section network based on LM algorithm. It can be illustrated on chart 1.

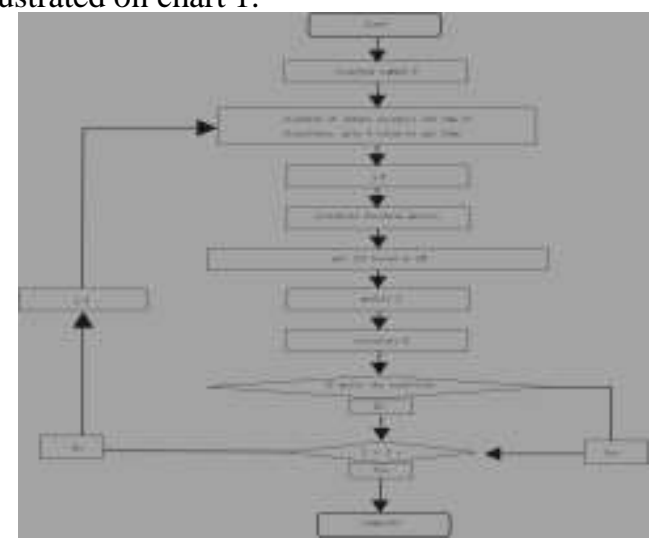

Chart 1 Basic flow chart of LM algorithm on neural network 
(1) Assign initial value of $\mathrm{W}$ as $\mathrm{W}(0)$, and iterations is $t=0$;

(2) Utilize learning sample $P$ to network and get relative output $Y$ through calculation, get the Marquardt signal of the last layer which can be propagated backward to get Jacobian matrix;

(3) Get modified value $\Delta X$ through connection value and threshold value based on LM algorithm

$$
\begin{aligned}
& \square X_{k}=-\left[J^{T}\left(X_{k}\right) J\left(X_{k}\right)+\mu_{k} I\right]^{-1} J^{T}\left(X_{k}\right) V\left(X_{k}\right) \\
& X(t)=X(t-1)+\Delta X
\end{aligned}
$$

Calculate performance function $E(X)$ to see if meet the condition.

If meet the condition, iteration is over. If not, $t+1 \rightarrow t$, to the second step to calculate for the second turn.

There are methods to measure if the iteration is completed or not. First, we could test the preset number of iteration is met. If so, we could complete calculation of iteration. Second, if the accuracy of iteration meet the qualification, namely, if the absolute value of performance function difference is less than the preset certain positive decimal. The last one, we could test if the average value of modified value $\Delta W$ connected with weight value is less than certain positive decimal.

\section{CALCULATIONS AND ANALYSIS}

\section{A. Choose sample and set parameter}

a. Choose sample of neural network

This paper utilizes time series of price and volume in Shanghai composite index ranging from 9th July 2012 to 24th September 2012. Data have been reformed to get 56 Phase point on the price index to do the rolling forecast. As BP neural network requires normalization, sample data is $x_{i}(i=1,2, \ldots, 56)$. Sample data is transferred into the range of $(-1,+1)$ based on the formula. The output data have been restored to get actual value.

b. Set parameter

Parameter setting of LM-BP: considering stability of prediction, this paper utilizes fixed weight and threshold values. Weight value from input layer to hidden layer is $0.5,0.75$ as weight value from hidden layer to output layer. Threshold values are 0.5 and 0 for hidden layer and output layer respectively. Range of hidden layer node is $[3,13]$. Reforming embedding dimension 26 is recognized as input dimension for LM-BP. This paper sets hidden node as 5 based on optimal hidden node algorithm. So the topology structure of 3 layer LM - BP neural network is 26-5-1.

Parameter setting of BP: training function utilizes gradient descent method with momentum. Learning function utilizes gradient descent function (Learndm). Learning efficiency is 0.4 . Transfer function is utilized between input layer to hidden layer is logarithmic function, linear function between hidden layer and output layer. Regarding to optimal algorithm of hidden node, this paper sets hidden node as 11 . So topology structure of this 3 layer BP neural network is 26-11-1.

\section{B. Analysis}

Utilize Matlab to programme to get LM-BP algorithm on PC Pentium4/CPU3.0GHz/RAM1.0G. To test the efficacy of this algorithm, LM-BP and BP models are utilized to predict Shanghai composite index respectively and then compared with the actual index later.

Evaluating indicator: evaluation of the prediction result uses the following indicator: normalized mean square error $v_{1}$, mean absolute difference $v_{2}$, and directional consistency index $v_{3}$. The first and second measure deviation between prediction value and actual value, the less they are, the more accuracy the prediction result is.

While, the third one could measure ratio of prediction, the bigger it is, the better it is.

Algorithm can be seen as follow:

$$
\begin{aligned}
& v_{1}=\frac{1}{\lambda^{2} n} \cdot \sum_{i=1}^{n}\left(p_{\text {real }, i}-p_{\text {pred }, i}\right)^{2} \\
& \lambda^{2}=\frac{1}{n-1} \cdot \sum_{i=1}^{n}\left(p_{\text {real }, i}-\overline{p_{\text {real }}}\right)^{2} \\
& v_{2}=\frac{1}{n} \cdot \sum_{i=1}^{n} \frac{\left|p_{\text {real }, i}-p_{\text {pred }, i}\right|}{p_{\text {real }, i}} \\
& v_{3}=\frac{100}{n} \cdot \sum_{i=1}^{n} d_{i}
\end{aligned}
$$

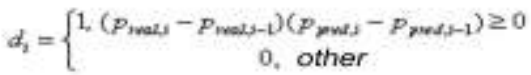

$$
\begin{aligned}
& p_{\text {real }, i} \text { is the actual closing price of the }
\end{aligned}
$$

day $i$ and $p_{\text {pred }, i}$ is the prediction closing

price $i=1,2, \ldots, n$.

TABLE I. COMPARISON BETWEEN LM-BP ALGORITHM AND BP ALGORITHM

\begin{tabular}{|l|l|c|c|c|c|}
\hline algorithm & $\begin{array}{c}\text { number } \\
\text { of } \\
\text { iteration }\end{array}$ & $\begin{array}{c}\text { Time } \\
\text { of } \\
\text { conver } \\
\text { gence } \\
\text { (s) }\end{array}$ & $v_{1}$ & $v_{2}$ & $v_{3}$ \\
\hline LM-BP & 11 & 2.58 & 0.163 & 0.022 & 53.57 \\
\hline BP & 2000 & 34.97 & 0.895 & 0.057 & 50.00 \\
\hline
\end{tabular}

As can be seen clearly from table 1, LM-BP presents superiority on both number of iteration and time of convergence and its prominent accuracy. ace them on the first page of your paper or as a footnote. 


\section{CONCLUSIONS}

This paper proposes prediction model of stock price on chaotic time series of multiple variable based on LMBP neural network. This model utilizes reformed multiple variables -embedding dimension 26 of, the time series of financial composite index as input dimension for LM-BP. The hidden node is set as 5 according to formula, output as the price of Shanghai Composite Index, and namely dimension 1. So network stability is 26-5-1. The experimental analysis firmly suggested that LM-BP is superior to BP neural network on accuracy and stability on prediction of Shanghai Composite index.

It is not necessary for LM-BP algorithm to hypothesize complicated, non-linear and instable financial system. LM-BP algorithm utilizes directly price of Shanghai Composite Index and reformed output and input data to simulate. LM-BP algorithm proposes new approach to study prediction issues of non-linear chaotic financial system. We could therefore conclude that it has theoretical and engineering application values.

\section{REFERENCES}

[1] W. L. Han, Non-linear analysis and prediction of Chinese stock market. Lanzhou: Northwestern Poly technical University, 2006.

[2] Y. $\mathrm{Xu}$ and T.Y. Jing, Identification and realization of neural network, Beijing: Electric Industry Press, 1999.

[3] X.Z. Bao, C. Liu, and B. Sun, "A Study on Setting Parameters of Stock Index Prediction by Applying LM-BP Neural Network", Journals of Systems and Management, Vol. 18. No . 6, Dec. 2009, pp.667-671.

[4] H.Y. Zhang \& Z. Geng, "New interpretation of LevenbergMarquart," Computer Engineering and Application, 2009, 45(19), pp.5-8.

[5] Z.H. Zhou \& Cao Cungen. Neural network and Application. Beijing: Tsinghua University Press, 2004.

[6] C.M. Hsu, "A hybrid procedure with feature selection for resolving stock/futures price forecasting problems," Neural Computing \& Applications, Vol. 22 Issue 3/4, Mar.2013, p651-671.doi: 10.1007/s00521-011-0721-4.

[7] J. Xiao and Z.L.Pan, "Stock price short-time prediction based on GA-LM-BP neural network", Journal of Computer Applications, vol. S1, 2012, pp.144-146.

[8] J.G. Lin, Investment in Securities, Beijing: Economy and Science Press, 2006.

[9] K.Cuthbetson, Quantitative Financial Economics: Stock, Bonds and Froeign Exchange. Wiley, 1996.

[10] H. Ince and T.B.Trafalis, "Short term forecasting with support vector machines and application to stock price prediction,'International Jounals of General Systems,vol.37, Issue 6, 2008,pp.677-687. doi:10.1080/03081070601068595. 